\title{
Method of real time simulation for design of microwave devices based on FDTD method
}

\author{
Hideki Kawaguchi $^{1 *}$ and Shohei Kodate ${ }^{2}$ \\ ${ }^{1}$ College of Information and Systems, Muroran Institute of Technology \\ ${ }^{2}$ Atmark Techno, Inc. \\ *kawa@mmm.muroran-it.ac.jp
}

Received: November 13, 2014; Accepted: May 13, 2015; Published: November 6, 2015

\begin{abstract}
This paper presents a method of a real time simulator for a computer aided design of microwave industrial products, in particular, for a shape design. According to progress of PC performance and simulation technology, numerical simulations are widely used in microwave product designs such as electromagnetic noise analysis, high frequency filter and so on. However, iterative microwave simulations for finding appropriate microwave product shapes require a large computer cost. It is shown that the real time microwave simulator based on the FDTD method can reduce a total simulation cost of the shape design to unify all stages of pre-process, calculation and post-process in the simulation.
\end{abstract}

Keywords: Computer simulation, Numerical methods

\section{Introduction}

A method of microwave simulation is widely used and essential tool not only in scientific researches but also in industrial product design such as estimation of electromagnetic noise from high frequency electric circuit, evaluation of S-parameters of microwave filter devices, and so on. In particular, the industry application of the microwave simulation itself is already big market and indeed there exist several commercial software tools on both of frequency and time domain methods. In general, such the numerical simulations of physical phenomena are carried out in three stages of pre-processing which is mainly constructed by numerical model making and discretization, calculation and post-processing like an animation of the simulation result. (Fig. 1) When the numerical simulation is used in any industrial product designs, we need to repeat the pre-processing and calculation stages many times to obtain appropriate shapes and parameters. The iteration of these stages is time consuming very much in principle, since the calculation stage is heavy task basically, and often 


\section{Journal of Advanced Simulation in Science and Engineering}

pre-processing as well. Otherwise, we need to employ any optimization schemes, such as generic algorithm (GA), particle swarm optimization (PSO), in which these stages are repeated automatically for finding optimized shapes or parameters. But it is impossible to watch detail of the optimization process and modify it timely in such the optimization schemes, and then we can obtain only final results.

This paper proposes a method of a real time simulation which is a unified process of three stages of pre-processing, calculation and post-processing all together for the microwave

\section{START}

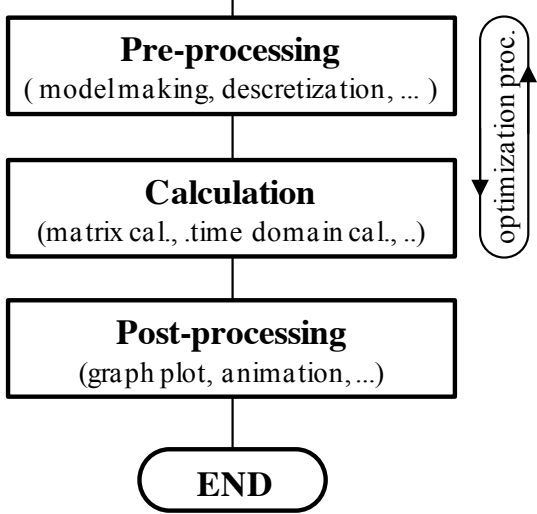

Figure 1: Overview of simulation flow product shape designs. In general, it takes long CPU time to obtain steady-state microwave field distribution, in addition, the shape design requires us to simulate for a lot of combinations of several parameters such as model sizes, material constants. This means that the shape design for the microwave products results in huge computation time, for example, a few days. In many cases, steady-state solutions for slightly different shapes have similar field distributions and characteristics each other. But we need to execute full simulations to find the final steady-state solutions for each iteration process in conventional simulations. Then, the real-time simulator, in which the three stages are unified all together, can directly make transition from one steady-state solution to others by changing the shape parameters during simulation with much shorter CPU time than conventional method. That is, turn-around-time between model making and calculation is effectively minimized, and one can find appropriate product parameters observing microwave field behavior numerically during design process by using the method of the real time simulation.

\section{FDTD microwave simulation}

We here adopt the finite difference time domain (FDTD) method for the microwave simulation which is one of the most reliable and popular schemes [1]. Although the FDTD scheme is quite well-known, we simply summarize its formulation for later reference with respect to the method of the real time simulation.

An entire grid space of the FDTD method and allocation of electromagnetic field components on the unit grid (Yee grid) are indicated in Fig. 2. For simplicity, it is assumed that medium are lossless and grid discretization is done by a uniform grid size $\Delta l$ for all directions. Then the formulation of the FDTD method is written by a central difference of Maxwell's equations as follows, 


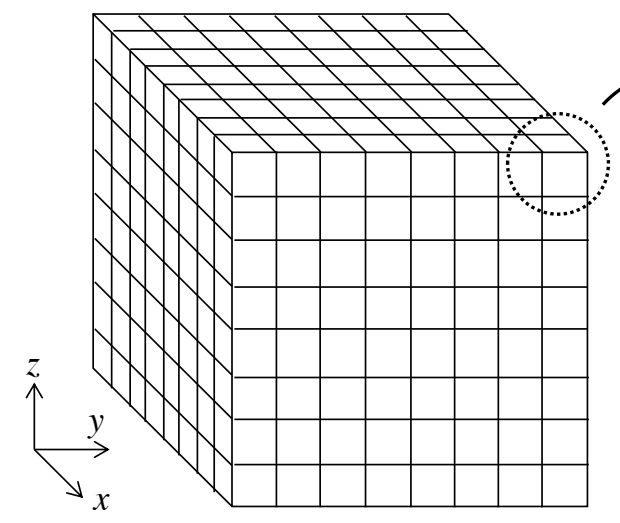

(a)

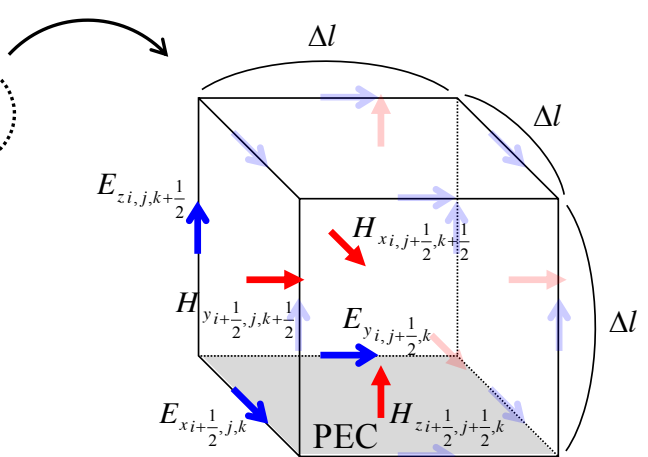

(b)

Figure 2: FDTD grid space (a) and field component allocation on unit grid (b)

$$
\begin{aligned}
& E_{x_{i+\frac{1}{2}, j, k}}^{n+1}=E_{x_{i+\frac{1}{2}, j, k}}^{n}+\frac{1}{\varepsilon_{i, j, k}} \frac{\Delta t}{\Delta l}\left(H_{z_{i+\frac{1}{2}, j+\frac{1}{2}, k}}^{n+\frac{1}{2}}-H_{z_{i+\frac{1}{2}, j-\frac{1}{2}, k}}^{n+\frac{1}{2}}-H_{y_{i+\frac{1}{2}}}^{n+\frac{1}{2}}+H_{y_{i+\frac{1}{2}}, j, k+\frac{1}{2}}^{n+\frac{1}{2}}\right) \\
& E_{y_{i, j+\frac{1}{2}, k}}^{n+1}=E_{y_{i, j+\frac{1}{2}, k}}^{n}+\frac{1}{\varepsilon_{i, j, k}} \frac{\Delta t}{\Delta l}\left(H_{x_{i, j+\frac{1}{2}, k+\frac{1}{2}}{ }^{n+\frac{1}{2}}}-H_{x_{i, j+\frac{1}{2}, k-\frac{1}{2}}{ }^{n+\frac{1}{2}}}-H_{z_{i+\frac{1}{2}, j+\frac{1}{2}, k}}^{n+\frac{1}{2}}+H_{z_{i-\frac{1}{2}, j+\frac{1}{2}, k}}^{n+\frac{1}{2}}\right)
\end{aligned}
$$

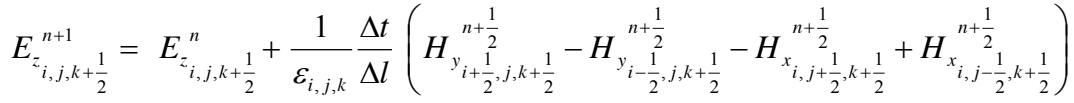

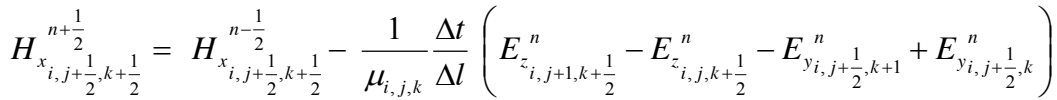

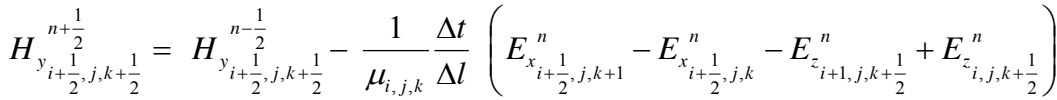

$$
\begin{aligned}
& H_{z_{i+\frac{1}{2}, j+\frac{1}{2}, k}^{n+\frac{1}{2}}}^{n}=H_{z_{i+\frac{1}{2}}, j+\frac{1}{2}, k}^{n-\frac{1}{2}}-\frac{1}{\mu_{i, j, k}} \frac{\Delta t}{\Delta l}\left(E_{y_{i+1, j+\frac{1}{2}, k}}^{n}-E_{y_{i, j+\frac{1}{2}, k}}^{n}-E_{x_{i+\frac{1}{2}, j+1, k}}^{n}+E_{x_{i+\frac{1}{2}, j, k}}^{n}\right)
\end{aligned}
$$

For a scatterer made by a perfect electric conductor (PEC), electromagnetic fields have to satisfy the following boundary condition on the PEC surface,

$$
\begin{aligned}
\mathbf{n} \times \mathbf{E} & =0 \\
\mathbf{n} \cdot \mathbf{H} & =0
\end{aligned}
$$

where $\mathbf{n}$ is a unit normal vector on the PEC surface. The boundary condition (2) means that tangential component of electric field $E_{/ /}$and normal component of magnetic field $H_{\perp}$ should vanish on the PEC. This situation is easily translated into the Yee grid, that is, when one plane of the Yee grid is PEC (see the bottom $x-y$ plane in Fig. 2(b)), $E_{/ /}$and $H_{\perp}$ com- $^{-}$ ponents, should be vanished, are just included in the PEC plane of the Yee grid. In other words, all field components on the PEC grid should be zero in the FDTD scheme.

In Fig. 3, an example of a simulation flowchart based on the FDTD method is indicated. The schemes of (1-1) and (1-2) are calculated for entire grid space and for all time steps. Then it should be noted that, owing to its time domain scheme, each components of (1-1) and 


\section{Journal of Advanced Simulation in Science and Engineering}

(1-2) can be calculated individually, that is, it is not necessary to solve any matrix equation, which is very important feature of the FDTD method. In addition, the boundary conditions (2) are also individually imposed on each component of fields, that is, the boundary condition setting can be overwritten after the field calculation (1-1) and (1-2) independently. For example, $H_{z}$ component calculation and the boundary condition setting can be performed as follows,

$$
\begin{aligned}
& H_{z_{i+\frac{1}{2}, j+\frac{1}{2}, k}}^{n+\frac{1}{2}}=H_{z_{i+\frac{1}{2}, j+\frac{1}{2}, k}}^{n-\frac{1}{2}}-\frac{1}{\mu_{i, j, k}} \frac{\Delta t}{\Delta l}\left(E_{y_{i+1, j+\frac{1}{2}, k}}^{n}-E_{y_{i, j+\frac{1}{2}, k}}^{n}-E_{x_{i+\frac{1}{2}, j+1, k}}^{n}+E_{x_{i+\frac{1}{2}, j, k}}^{n}\right) \\
& H_{z_{i+\frac{1}{2}, j+\frac{1}{2}, k}}^{n+\frac{1}{2}}=H_{z_{i+\frac{1}{2}, j+\frac{1}{2}, k}}^{n-\frac{1}{2}} \times i g d_{i+\frac{1}{2}, j+\frac{1}{2}, k}
\end{aligned}
$$

or

$$
H_{z_{i+\frac{1}{2}, j+\frac{1}{2}, k}}^{n+\frac{1}{2}}=\left\{H_{z_{i+\frac{1}{2}, j+\frac{1}{2}, k}}^{n-\frac{1}{2}}-\frac{1}{\mu_{i, j, k}} \frac{\Delta t}{\Delta l}\left(E_{y_{i+1, j+\frac{1}{2}, k}}^{n}-E_{y_{i, j+\frac{1}{2}, k}}^{n}-E_{x_{i+\frac{1}{2}, j+1, k}}^{n}+E_{x_{i+\frac{1}{2}, j, k}}^{n}\right)\right\} \times i g d_{i+\frac{1}{2}, j+\frac{1}{2}, k}
$$

where

$$
i g d_{i+\frac{1}{2}, j+\frac{1}{2}, k}= \begin{cases}0 & \text { for PEC grid } \\ 1 & \text { for non - PEC grid }\end{cases}
$$

Accordingly, to appropriately set $\operatorname{igd}_{i+1 / 2, j+1 / 2, k}$ values for all grids in advance with the FDTD calculation, the PEC boundary condition is automatically imposed by (3).

\section{Real time FDTD simulation}

As an example, we here use a microwave propagation problem which consists of a cavity and a power input waveguide. That is, a two-dimensional numerical model and electric field distribution in a steady-state, which is calculated by the FDTD method, are indicated in Fig. 4, and a time domain behavior of total energy inside the cavity is shown in Fig. 5. In the case of industrial applications, we interested in mainly steady-state results of the field distribution, reflection coefficient, and so on. But we need to execute the FDTD calculation for over several hundred periods (several 10,000 time steps in Fig. 5) for obtaining the steady-state results in cases of high Q (quality factor) cavities. In addition, we

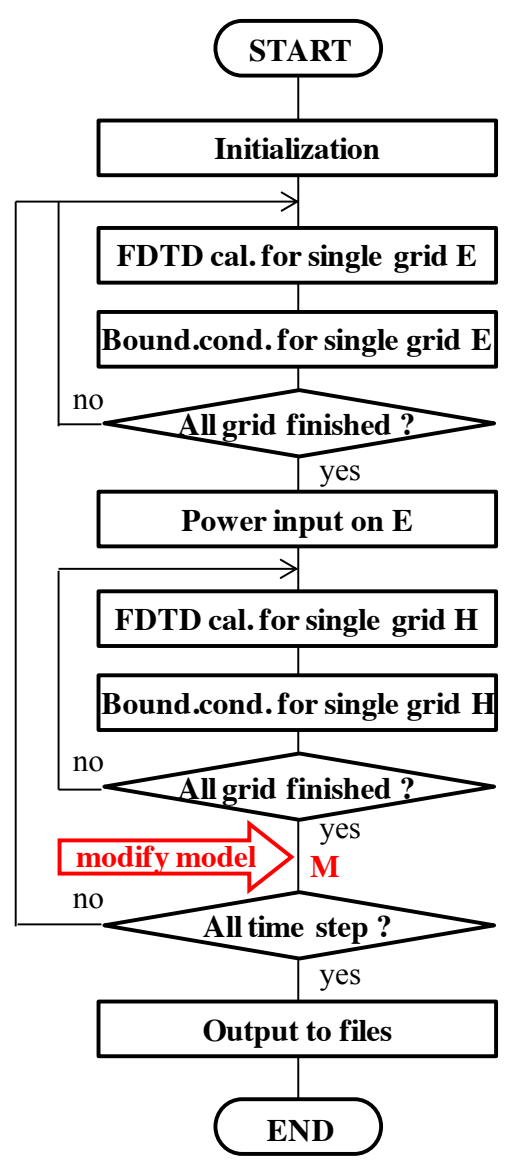

Figure 3: Example of FDTD flowchart 


\section{Journal of Advanced Simulation in Science and Engineering}

have to repeat this long calculation for every numerical model sizes or shapes, material constants, and other numerical parameters in the design process. (see Fig. 6(a)) This is just main reason why the shape design of microwave products by using the FDTD microwave simulation takes very long CPU time.

Then, we find that most part of long calculation time for the steady-state in the design process are not necessary, and entire calculation time in the design process can be reduced effectively to take into account the non-simultaneous

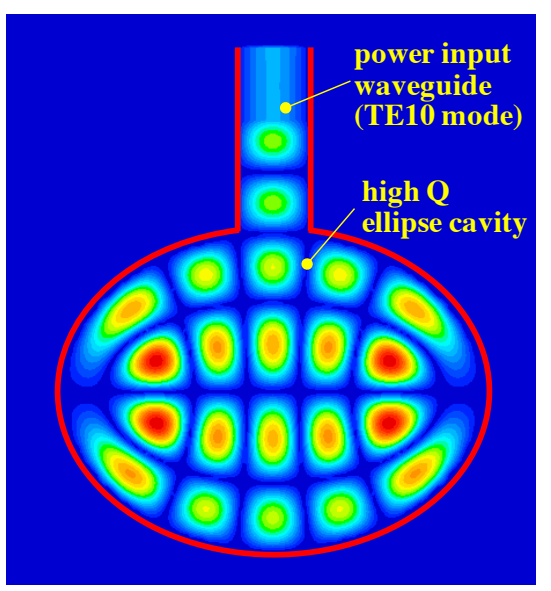

Figure 4: Microwave cavity and waveguide properties of the FDTD scheme as in (3). (see Fig. 6(b)) That is, once we obtain the steady-state field distribution by the FDTD calculation after several 10,000 time steps, other steady-state solutions can be obtained within much shorter time steps (typically several 100

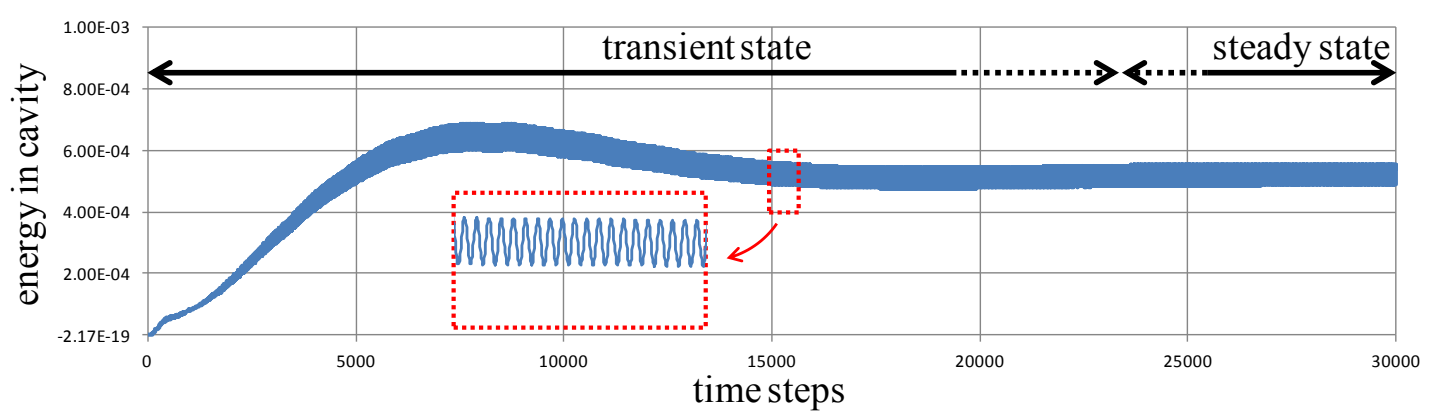

Figure 5: Time domain behavior of electromagnetic field energy inside cavity

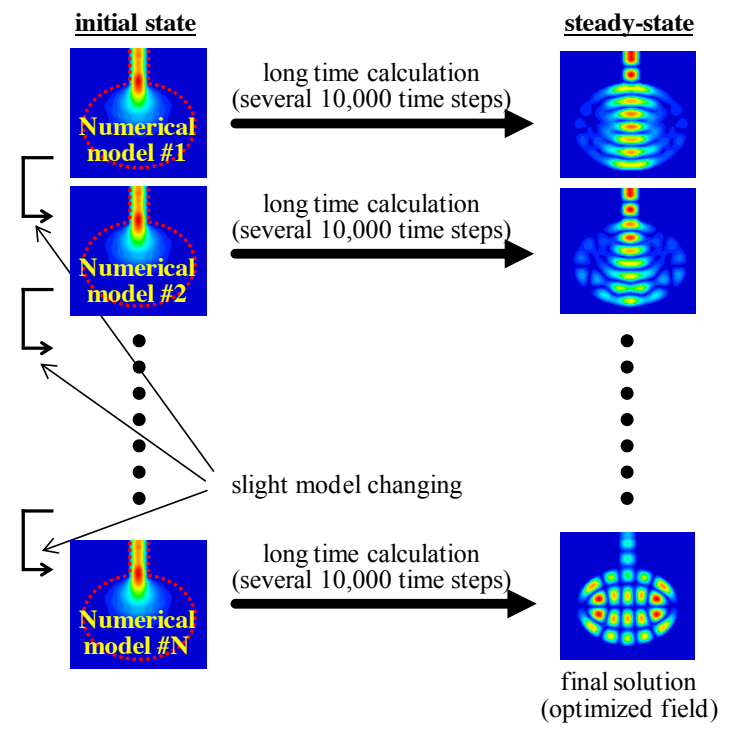

(a) conventional iteration process

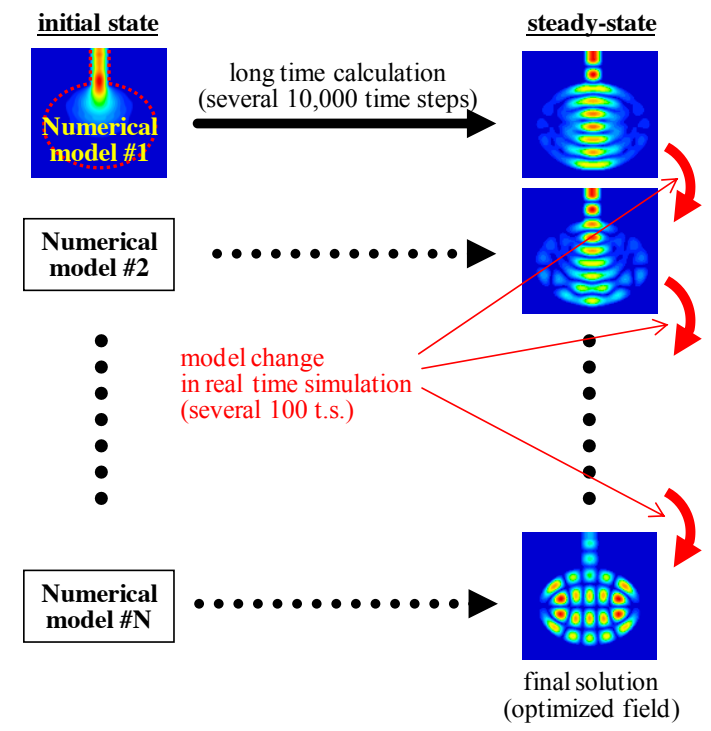

(b) method of real time simulation

Figure 6: Calculation costs in shape design process 


\section{Journal of Advanced Simulation in Science and Engineering}

time steps) by changing the numerical model in real time at the timing marked " $\mathrm{M}$ " in the FDTD flowchart of Fig. 3, since field distributions for slightly different numerical models may be similar each other. That is, the numerical model is changed interrupting at the timing just after the FDTD scheme calculations of electric and magnetic fields for all grid space are finished and just before the next time step calculation will start (without stopping program), which means that the previous time step field distribution will be used as the initial value of the next time step calculation for the modified numerical model. Accordingly most of the calculation time for the steady-state (for the numerical models of \#2 - \#N in Fig. 6(b)) is reduced by this method. Then change of the numerical model should be done by hand to achieve required characteristics of the microwave product, watching the behavior of the microwave field carefully. Therefore this real time simulation has to be executed by the unified system of the pre-processing, calculation and the post-processing.

The FDTD method is a transient (time domain) analysis in principle, and, in general, the FDTD transient simulation reaches to the steady-state after several hundred periods. Then the steady-state is a kind of solution to frequency domain analysis of the cavity, therefore the field distribution should be unique basically and independent of intermediate history during the transient simulations in Fig. 6 (b) except for degeneration cases.

\section{Numerical example}

At the final part of this paper, a dedicated application software developed by Microsoft Visual $\mathrm{C}++$ tool is shown as an example of the method of the real time simulator for the shape design of microwave products. The dedicated application software is made for finding appropriate shape of a outlet part of a microwave heating device which consists of the TE10 mode waveguide and a high Q cavity with elliptic shape. (Fig. 7) It is assumed that the microwave heating device is used to heat a asphalt pavement with damages for its repairing [2]. The high Q cavity is inserted near the microwave outlet to isolate the wave-

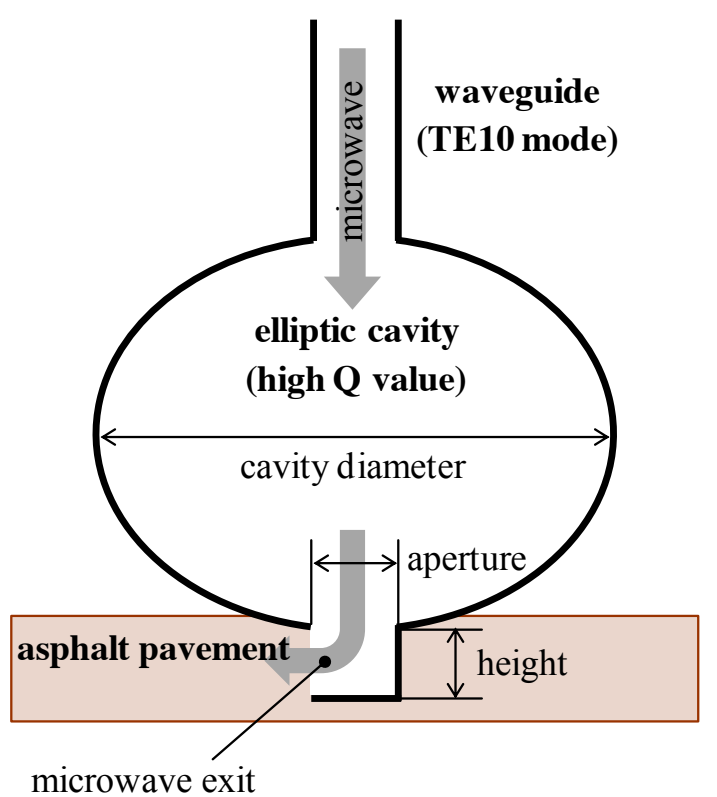

Figure 7: Outlet of microwave heating device guide and the exit side each other, and to provide stable injection of microwave power to various conditions of the asphalt pavement. Then, the impedance matching of the micro- 


\section{Journal of Advanced Simulation in Science and Engineering}

wave injection strongly depends on the exit shape of the elliptic cavity and there are too many possibilities in design of the cavity shape. For example, when we consider 10 cases for sizes of the cavity diameter, the height and the aperture width of the exit part, we need to execute simulations for 1,000 numerical models if we employ conventional design process of Fig. 6(a). That is, the shape design by the FDTD simulations requires huge computational cost, in general.

In the dedicated application software indicated in Fig. 8, the scheme of Fig. 6(b) is implemented. The two-dimensional FDTD microwave simulation will start simply by pressing "START" button from a zero microwave field situation. At the left-upper region, the electric field distribution (contour lines for field intensity) is displayed and updated in real time for every period. The red and blue colors indicate positive and negative values. In the left-lower graph, time domain signals of reflection (red color) and transmitting (blue color) ratio, which are calculated by the Poynting vector at the waveguide, are plotted, and the time domain behavior of total amount of microwave field energy inside the elliptic cavity is shown in the right-lower graph. To use these time domain signal plots, we can understand the global energy flow in this microwave device, and can judge if the simulation reaches to

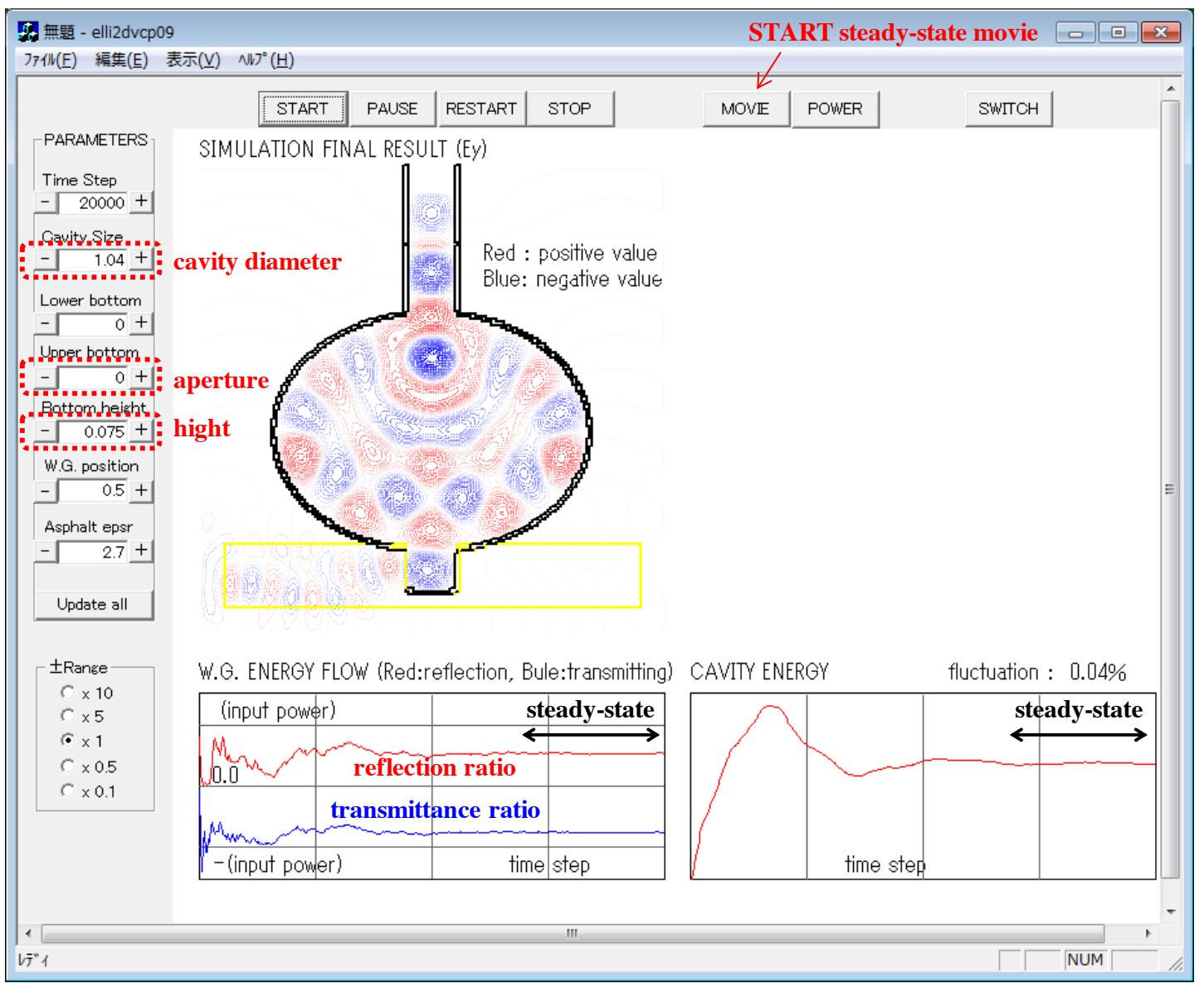

Figure 8: Example of execution of real time simulator 


\section{Journal of Advanced Simulation in Science and Engineering}

steady-state. (see Fig. 8) The horizontal axes of the left and right - lower graphs are normalized by the maximum time step. The vertical axis of the left-lower graph is scaled by the input microwave power and that of the right-lower graph is normalized by the maximum cavity energy in real-time. In this simulator, the numerical model of the elliptic cavity is defined in $180 \times 200$ grid space for all shapes. That is, the numerical model shape is expressed by specifying the individual grid properties (PEC, vacuum, dielectric material, and so on) using the $2 \mathrm{D}$ array of $i g d_{i+1 / 2, j+1 / 2, k}$ in (3). Then, changing of the numerical model is done by using the vertical series of edit boxes in the left region. In the edit boxes, parameters of the numerical model such as the cavity diameter, the height and aperture width of the exit part are displayed, and these parameters can be changed anytime during the real time simulation. And then, grid information values of $i g d_{i+1 / 2, j+1 / 2, k}$ (used in (3)) are updated by using these numerical model parameters at the timing of "M" in the FDTD calculation flowchart (Fig. 3). Accordingly, change of numerical model can be carried out in the real time simulation as in Fig. 6(b).

In this shape design, we want to find the shape for the best heating efficiency, that is, the main task is to decide a parameter set of the cavity diameter, sizes of the height and the aper-

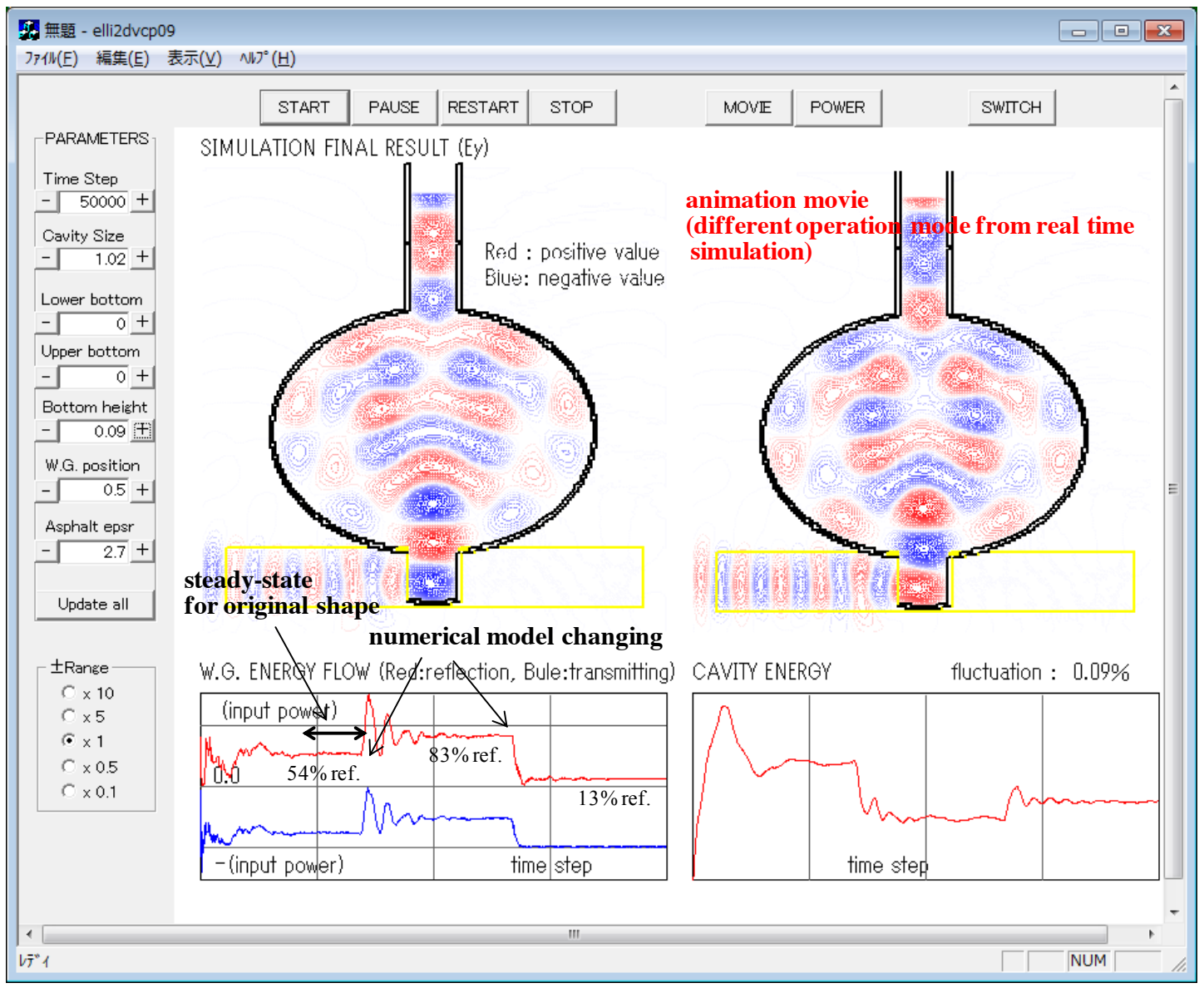

Figure 9: Example of shape design operation of real time simulator 


\section{Journal of Advanced Simulation in Science and Engineering}

ture width of the exit part which results in the lowest reflection ratio or the highest transmittance ratio, referring the time domain signals in the left-lower graph. In Fig. 9, an example of the shape design process based on this real time simulator is shown. After reaching the steady-state for the original numerical model, we can see two discontinuities in the left-lower graph of the energy flow signal, which indicate changing of the numerical model during the real time simulation. Then the original reflection ratio of $54 \%$ is improved to $13 \%$ which is acceptable value in practical device operation. It is imagined that the improvement of the shape of the microwave hearing device is performed very efficiently by this method of the real time simulator compared with iteration of full simulations for each numerical model. In addition, a steady-state behavior of the final solution can be confirmed by an animation movie of repeated displaying the field distribution for one period at the left-upper region. It is also confirmed that the field distribution and reflection ratio of Fig. 9 is independent of intermediate calculations by executing calculations for various cases of parameter changing for the same parameters as in Fig. 9. As an example, a simulation for the same parameters as in Fig. 9 with different intermediate calculations is indicated in Fig. 10. Strictly speaking, there exist about $1 \%$ error in the final reflection ratios between Fig. 9 and Fig. 10, but both

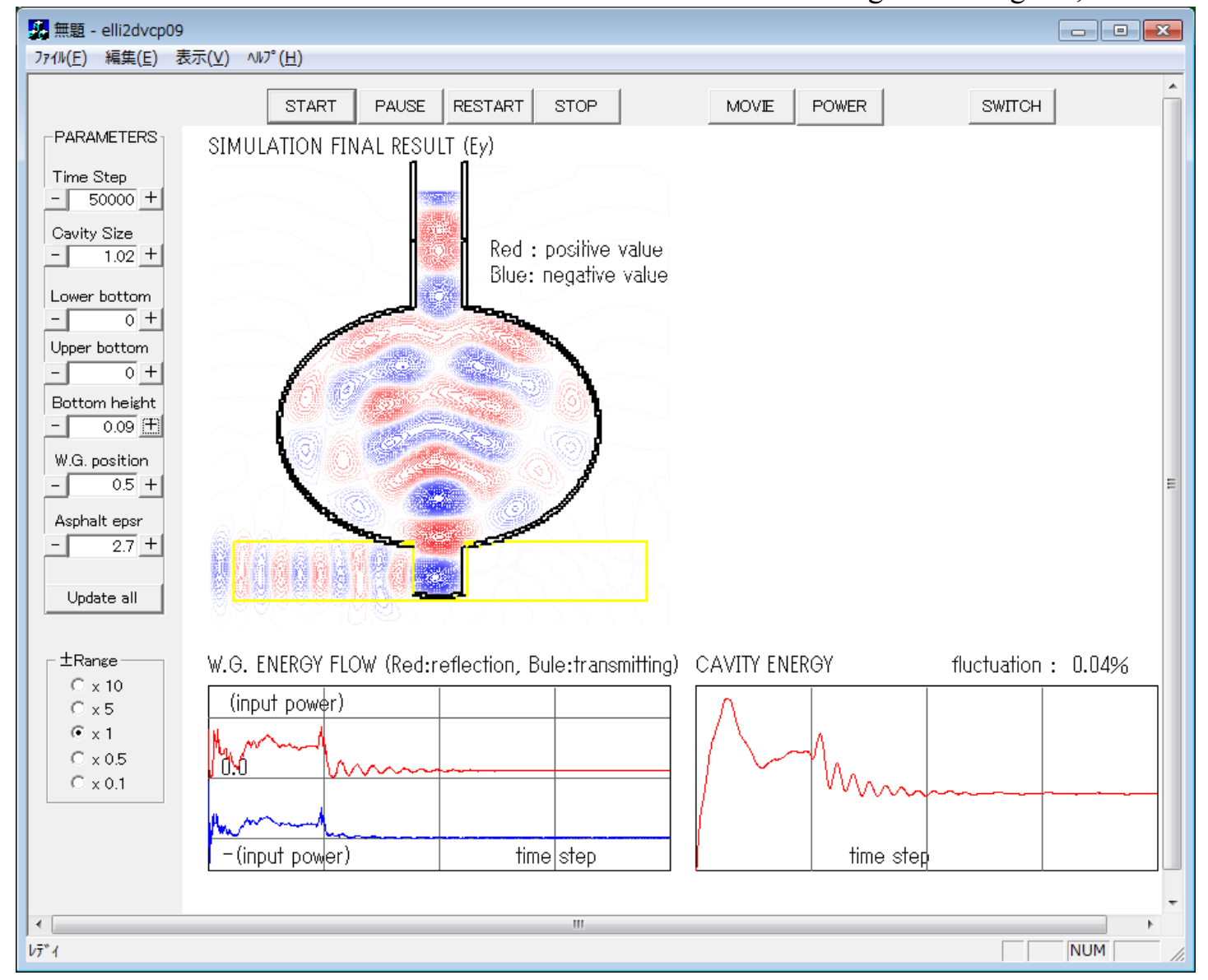

Figure 10: Example of different design process for same final parameter as Fig. 9 


\section{Journal of Advanced Simulation in Science and Engineering}

\section{Summary}

In this paper, a method of a real time simulator for efficient shape design of microwave devices has been proposed, and the dedicated application software of the microwave heating device has been shown as an example of the real time simulator. The presented dedicated application software can be used only for the specialized problem of the microwave heating device, but the concept of the shape design based on the real time simulator can be applied to wider kinds of problems. In particular, the dedicated application software itself is easily converted for other microwave simulations by changing values of the grid information $i g d_{i+1 / 2}$, $j+1 / 2, k$ and allocations of numerical model parameters to design parameters which are displayed in the left region of Fig. 8.

It is noticed that an essential advantage of the presented methodology of the shape design by the real time simulator is "real time designing" in which we can observe all process of the design and can change the numerical model as the user wants. For example, a combination of a frequency domain method based on the FEM and any kinds of optimization schemes such as GA, PSO will give us efficient shape optimization indeed. But we can obtain only one optimized result from such the automatic optimization methods and there are no choices by the users from various viewpoints.

Finally, we have to refer to the two-dimensional simulation in the presented dedicated application software. In general, most of practical microwave problems belong to three-dimensional phenomena, that is, the presented dedicated application software can be used to only limited microwave problems (i.e. two-dimensional) by changing the grid information. The reason why this paper treat two-dimensional problems is to reduce calculation time including the FDTD calculation and the animation as the post-processing. For example, a method of a high-speed visualization of the FDTD simulation using the GPU is proposed by Okubo at.al. [3] The use of this method may be one of solutions to three-dimensional version of the method of the real time simulator.

\section{References}

[1] A. Taflove, S. C. Hagness: Computational Electrodynamics: The Finite-Difference Time-Domain Method (2nd Ed.), Artech House, Norwood, 2000.

[2] S. Kagami, M.Sakuraba, T.Yano, H.Saitoh, H.Kawaguchi: Microwave-Assisted Surface Recycling of Asphalt Pavement, Proc. of MAGDA Conf. in Sapporo (Conf. on Electromagnetic Phenomena and Dyanamics), (2010), 185-188. (in Japanese)

[3] N. Kawada, K. Okubo, N. Tagawa, T. Tsuchiya: High-speed Visualization for GPU-FDTD Simulation of Electromagnetic Field Using CUDA and OpenGL, EMT-10-141 (2010), 49-54. (in Japanese) 